\title{
Experienced, Recreational Scuba Divers in Australia Continue to Dive Despite Medical Contraindications
}

\author{
David McD. Taylor, MD; Kevin S. O’Toole, MD; Christopher M. Ryan, PhD \\ From the Director of Emergency Medicine Research, Royal Melbourne Hospital, Victoria, Australia (Dr Taylor); and the Department of \\ Emergency Medicine (Dr O'Toole), and the Western Psychiatric Institute \& Clinic (Dr Ryan), University of Pittsburgh, Pittsburgh, PA.
}

\begin{abstract}
Objective.-In Australia, a medical examination is required before undertaking a scuba diving course in order to screen for contraindications to diving. No further medical screening is required, and yet divers may develop diseases during their diving careers. This study aimed to survey experienced recreational scuba divers to determine the prevalence of diseases contraindicated in diving.

Methods.-A cross-sectional, postal survey was taken of divers belonging to scuba diving clubs across Australia.

Results. - Three hundred forty-six divers returned completed questionnaires. Two hundred fiftyfour $(73.4 \%)$ were male, and $258(74.6 \%)$ were aged 31 to 60 years. The mean years of diving equaled $10.6 \pm 9.18$ years, and the mean number of dives undertaken was $414 \pm 740$ dives. One hundred sixty-two (46.8\%) divers were overweight, 45 (13.0\%) divers required regular medication, and 39 $(11.3 \%)$ divers smoked. Thirty-six (10.4\%) divers reported a past or present history of asthma, and the same number reported hypertension or coronary heart disease. Eighty-six (24.9\%) divers reported past or present psychological symptoms. Forty-two (12.1\%) divers reported hearing difficulties, and $81(23.4 \%)$ divers reported past or present tinnitus. Two divers had a past history of epilepsy, 2 had a history of pneumothorax, and 1 was diabetic.

Conclusions.-Experienced, recreational scuba divers continue to dive despite medical contraindications. This raises the questions: Did the divers fail to disclose these conditions at the initial examination, or did these conditions develop subsequently? Is the risk associated with these conditions clinically significant, and should screening examinations be undertaken at regular intervals? The high prevalence of hearing difficulties and tinnitus may be the result of aural barotrauma and requires further research.
\end{abstract}

Key words: scuba, diving, medical examination

\section{Introduction}

Scuba diving is a popular but high-risk sport. ${ }^{1,2}$ In Australia, medical practitioners screen diving candidates for medical conditions thought to increase the risks of diving. 1,3 Today, standards for fitness to scuba dive are described in the Australian Standards, ${ }^{4}$ and guidelines for medical examinations have been published., ${ }^{1,2}$

This research was presented at the 2001 Annual Scientific Meeting of the Australasian College for Emergency Medicine, Hobart, Australia, October 1, 2001.

The authors declare that no conflict of interest exists regarding this research

Corresponding author: David McD. Taylor, MD, Emergency Department, Royal Melbourne Hospital, Grattan St, Parkville, Victoria, Australia 3050 (e-mail: david.taylor@mh.org.au). Offprints will not be available from the author.
Traditionally, candidates with medical conditions contraindicated in diving (eg, asthma, diabetes, and epilepsy) have been excluded from the sport via the medical examination. However, the screening medical examination may not detect such conditions, ${ }^{6}$ and the practitioner's determination of fitness to dive may be inconsistent. ${ }^{7}$ Also, anecdotal and some published evidence suggests that divers with contraindicated conditions tend to remain covert and may falsify their medical history in order to dive. ${ }^{6,8}$

In Australia, data on the prevalence of contraindicated conditions among divers are scant. In particular, the fitness to dive of experienced recreational divers is not known. Importantly, this group may have been screened and certified as fit at the beginning of their diving careers 
Table 1. Sex and age of the subjects $(n=346)$

\begin{tabular}{lrrrrrrr}
\hline \multicolumn{7}{c}{ Subject age group $(y)$} \\
\cline { 2 - 8 } & $18-20$ & $21-30$ & $31-40$ & $41-50$ & $51-60$ & $61+$ & Totals \\
\hline Male & $5(1.5 \%)$ & $44(12.7 \%)$ & $85(24.6 \%)$ & $69(19.9 \%)$ & $46(13.3 \%)$ & $5(1.5 \%)$ & $254(73.4 \%)$ \\
Female & $5(1.5 \%)$ & $28(8.1 \%)$ & $35(10.1 \%)$ & $16(4.6 \%)$ & $7(2.0 \%)$ & $1(0.3 \%)$ & $92(26.6 \%)$ \\
Totals & $10(2.9 \%)$ & $72(20.8)$ & $120(34.7 \%)$ & $85(24.6 \%)$ & $53(15.3 \%)$ & $6(1.7 \%)$ & $346(100 \%)$ \\
\hline
\end{tabular}

but may have developed contraindications that have subsequently placed them at increased risk.

This study aimed to examine the health of experienced, recreational scuba divers in Australia and to determine the prevalence of medical conditions that preclude diving within this group. These data will assist in evaluating the effectiveness of the initial medical screening process, the need for repeated medical examinations, and the safety of these conditions among experienced, recreational divers.

\section{Methods}

The study was a cross-sectional survey of active, experienced scuba divers belonging to scuba diving clubs across Australia. A list of clubs, published on the "Diving in Australia" Internet website, ${ }^{9}$ was used to identify sources of potential subjects. E-mail or telephone contact with each club's contact person (president or secretary) was attempted. Forty-eight clubs were contactable, and all agreed to participate in the study.

In June 2000, each club's contact person was mailed a variable number of study questionnaires depending on the number of active members. These were distributed at the next club meeting, completed immediately, and returned by the contact person in a stamped, addressed envelope. Two months after the initial mailing, clubs that had not returned questionnaires were mailed a reminder letter. This was repeated in 1 month's time, when necessary.

The study questionnaire was anonymous and designed by the authors. The first section collected demographic data on the divers, including diving experience. The second section consisted of a list of medical conditions, each with the potential to interfere with a diver's activity and potentially compromise safety. This list was derived from published sources of information relating to medical fitness to dive. ${ }^{1,3-5}$ The divers were asked to record when they had ever experienced each of the conditions. The possible responses were "never," "in the past," and "now and I dive with it." Only 1 response was permitted for each condition.
The body mass index (BMI) was calculated by the investigators on the basis of reported weight and height values. The following classifications were used: BMI $<20$ (underweight), 20 to 24.9 (normal), 25 to 29.9 (overweight), 30 to 34.9 (obese), and $\geq 35$ (morbid obesity).

Most responses were analyzed descriptively. Mean values are reported with SDs. Comparisons of proportions used the Yate's corrected chi-square test with EpiCalc, version 1.02 (2000) software.

The study was authorized by the Institutional Review Board (Ethics Committee) of the University of Pittsburgh, PA.

\section{Results}

Of the 48 clubs that initially agreed to participate, 29 $(60.4 \%)$ ultimately returned questionnaires (New South Wales 10, Victoria 7, Western Australia 4, Northern Territory 3, South Australia 2, Tasmania 2, and Queensland 1). A total of 346 questionnaires were returned.

The divers' sex and age are described in Table 1. Almost three quarters were male, and most (59.3\%) were middle aged (31-50 years). As expected, most were experienced scuba divers. The mean "years of diving" was $10.6 \pm 9.18$ years (median, 7.5), and the mean "number of dives done" was $414 \pm 740.82$ dives (median, 200).

Table 2 describes factors associated with the divers' socioeconomic status. Most divers were university graduates or postgraduates (206 divers, 59.6\%) and were in white collar or professional employment (208 divers, $60.1 \%$ ).

Table 3 describes the divers' risk factors for illness. Almost half were classified as overweight or greater (162 divers, 46.8\%). Importantly, 8 divers $(2.3 \%)$ were morbidly obese. Most divers admitted to a small $(=5$ drinks per week) or moderate alcohol intake (6-10 drinks per week), with only 42 divers (12.1\%) consuming more than 15 drinks per week. The number of smokers in the group was relatively small, and approximately the same number reported illicit drug use (marijuana, 
Table 2. Socioeconomic status of patients (percentages in parentheses)

\begin{tabular}{lrrlrr}
\hline \multicolumn{2}{c}{ Highest level of education completed } & \multicolumn{3}{c}{ Employment status } \\
\hline Primary school & 8 & $(2.3)$ & Unemployed & 24 & $(6.9)$ \\
High school & 131 & $(37.9)$ & Blue collar/trade & 104 & $(30.1)$ \\
University & 157 & $(45.4)$ & White collar/clerical & 53 & $(15.3)$ \\
Postgraduate & 49 & $(14.2)$ & Professional & 155 & $(44.8)$ \\
No response & 1 & $(0.3)$ & No response & 10 & $(2.9)$ \\
Totals & 346 & $(100)$ & & 346 & $(100)$ \\
\hline
\end{tabular}

amphetamines, and LSD). Only 53 divers reported taking daily medication.

Table 4 describes the medical conditions experienced by some divers that are generally regarded as relative or absolute contraindications to diving.

Table 3. Risk factors for illness among the divers $(n=346$; percentages in parentheses)

\begin{tabular}{|c|c|c|}
\hline & \multicolumn{2}{|c|}{ Risk factors (\%) } \\
\hline \multicolumn{3}{|l|}{ The body mass index (BMI): } \\
\hline Underweight $(<20)$ & 9 & $(2.6)$ \\
\hline Normal (20-24.9) & 157 & $(45.4)$ \\
\hline Overweight (25-29.9) & 118 & $(34.1)$ \\
\hline Obese $(30-34.9)$ & 36 & $(10.4)$ \\
\hline Morbid obesity ( $\geq 35$ ) & 8 & $(2.3)$ \\
\hline No response & 18 & $(5.2)$ \\
\hline \multicolumn{3}{|c|}{ No. of alcoholic drinks per week: } \\
\hline None & 28 & $(8.1)$ \\
\hline$<1$ & 52 & $(15.0)$ \\
\hline $1-5$ & 120 & $(34.7)$ \\
\hline $6-10$ & 62 & $(17.9)$ \\
\hline $11-15$ & 40 & $(11.6)$ \\
\hline $15+$ & 42 & $(12.1)$ \\
\hline No response & 2 & $(0.6)$ \\
\hline \multicolumn{3}{|l|}{ Smoking status: } \\
\hline Nonsmoker & 294 & $(85.0)$ \\
\hline Smoker & 39 & $(11.3)$ \\
\hline No response & 13 & $(3.8)$ \\
\hline \multicolumn{3}{|l|}{ Illicit drug use: } \\
\hline Not used & 303 & $(87.6)$ \\
\hline Used* & 37 & $(10.7)$ \\
\hline No response & 6 & $(1.7)$ \\
\hline \multicolumn{3}{|l|}{ Daily medication use: } \\
\hline $\mathrm{OCP} \dagger$ only & 8 & $(2.3)$ \\
\hline OCP plus other medication & 1 & $(0.3)$ \\
\hline Other medication & 44 & $(12.7)$ \\
\hline
\end{tabular}

*Marijuana, 28; amphetamines, 3; LSD, 2; not specified, 4. $\dagger \mathrm{OCP}$ indicates oral contraceptive pill.
Respiratory and upper respiratory diseases predominated. Seasonal allergies were common, with almost half of all divers having either a history of allergies or an active disease. Importantly, 28 divers $(8.1 \%)$ had a history of asthma or chronic obstructive airways disease, and an additional 10 divers (2.9\%) were current sufferers. As expected, pressure equalizing problems were common, with 50 divers $(14.5 \%)$ continuing to dive despite this difficulty. Tympanic membrane rupture was not uncommon. However, as with the 2 cases of round or oval window rupture, it is not known if these injuries were caused by barotrauma while scuba diving. Also of importance is the finding that 2 divers reported a history of pneumothorax.

A history of neurological conditions was reasonably common, although prevalent illness was confined mainly to recurrent headaches (19 divers, 5.5\%) and migraine (13 divers, 3.8\%). No diver reported suffering from epilepsy at the time of the survey, although 2 divers had a past history of this condition. Single divers reported a history of brain tumor, transient ischemic attack, and brain aneurysm, and 5 divers reported a history of significant head trauma. Thirteen divers $(4.8 \%)$ reported having suffered from decompression illness. Cardiac conditions were relatively uncommon and mainly confined to hypertension (12 divers, 3.5\%). It is possible that some divers who reported a history of hypertension were, in fact, being treated for this condition. Importantly, 3 divers reported a history of angina. The nature of the cardiac arrhythmias reported is unknown. Many divers reported a history of psychiatric illness and, importantly, 14 divers $(4.0 \%)$ continued to dive with either claustrophobia or anxiety. Only 1 diver suffered from diabetes at the time of the survey.

Table 5 describes the medical conditions experienced by some divers that are not generally regarded as relative or absolute contraindications to diving. However, these conditions may interfere with a diver's activity or may be aggravated by diving. Tinnitus was surprisingly common, with almost one quarter of all divers having a history of or presently suffering from this condition. Hear- 
Table 4. Reported medical conditions that are relative and absolute contraindications to diving $(n=346$; percentages in parentheses)

\begin{tabular}{|c|c|c|c|c|c|c|}
\hline \multirow[b]{2}{*}{ Disease system } & \multicolumn{3}{|c|}{ Past history of disease } & \multicolumn{3}{|c|}{ Presently suffers from disease } \\
\hline & Male & Female & Total & Male & Female & Total \\
\hline \multicolumn{7}{|l|}{ Respiratory: } \\
\hline Seasonal allergies & $54(15.6)$ & $18(5.2)$ & $72(20.8)$ & $54(15.6)$ & $25(7.2)$ & $79(22.8)$ \\
\hline Asthma & $21(6.1)$ & $6(1.7)$ & $27(7.8)$ & $5(1.5)$ & $4(1.2)$ & $9(2.6)$ \\
\hline Chronic obstructive airways disease & $\ldots$ & $1(0.3)$ & $1(0.3)$ & $1(0.3)$ & $\ldots$ & $1(0.3)$ \\
\hline Spontaneous pneumothorax & $\ldots$ & $1(0.3)$ & $1(0.3)$ & $\ldots$ & $\ldots$ & $\ldots$ \\
\hline Traumatic pneumothorax & $1(0.3)$ & $\ldots$ & $1(0.3)$ & $\ldots$ & $\ldots$ & $\ldots$ \\
\hline \multicolumn{7}{|l|}{ Eye, ear, nose, and throat: } \\
\hline Equalizing problems in the ears or sinuses & $68(19.7)$ & $22(6.4)$ & $90(26.0)$ & $31(9.0)$ & $19(5.5)$ & $50(14.5)$ \\
\hline Chronic sinusitis & $9(2.6)$ & $1(0.3)$ & $10(2.9)$ & $7(2.0)$ & $5(1.5)$ & $12(3.5)$ \\
\hline Diving within 12 mo of eye surgery & $5(1.5)$ & $2(0.6)$ & $7(2.0)$ & $2(0.6)$ & $\ldots$ & $2(0.6)$ \\
\hline Chronic otitis media & $10(2.9)$ & $2(0.6)$ & $12(3.5)$ & $1(0.3)$ & $\ldots$ & $1(0.3)$ \\
\hline Ear drum rupture & $17(4.9)$ & $4(1.2)$ & $21(6.1)$ & $\ldots$ & $\ldots$ & $\ldots$ \\
\hline Inner ear surgery & $5(1.5)$ & $\ldots$ & $5(1.5)$ & $\ldots$ & $\ldots$ & $\ldots$ \\
\hline Round or oval window rupture & $2(0.6)$ & $\ldots$ & $2(0.6)$ & $\ldots$ & $\ldots$ & $\ldots$ \\
\hline Penetrating eye injury & $1(0.3)$ & $\ldots$ & $1(0.3)$ & $\ldots$ & $\ldots$ & $\ldots$ \\
\hline \multicolumn{7}{|l|}{ Neurological: } \\
\hline Recurrent headaches & $36(10.4)$ & $14(4.1)$ & $50(14.5)$ & $10(2.9)$ & $9(2.6)$ & $19(5.5)$ \\
\hline Migraine headaches & $38(11.0)$ & $18(5.2)$ & $56(16.2)$ & $5(1.5)$ & $8(2.3)$ & $13(3.8)$ \\
\hline Brain or spinal cord damage (disease or trauma) & $5(1.5)$ & $\ldots$ & $5(1.5)$ & $3(0.9)$ & $\ldots$ & $3(0.9)$ \\
\hline Spinal cord injury (no permanent damage) & $5(1.5)$ & $\ldots$ & $5(1.5)$ & $2(0.6)$ & $\ldots$ & $2(0.6)$ \\
\hline Decompression sickness (with nerve damage) & $10(3.9)$ & $2(0.6)$ & $12(3.5)$ & $1(0.3)$ & $\ldots$ & $1(0.3)$ \\
\hline Brain tumor & $\ldots$ & $1(0.3)$ & $1(0.3)$ & $\ldots$ & $1(0.3)$ & $1(0.3)$ \\
\hline Epilepsy & $1(0.3)$ & $1(0.3)$ & $2(0.6)$ & $\ldots$ & $\ldots$ & $\ldots$ \\
\hline Head trauma (unconscious $>24 \mathrm{~h}$ ) & $4(1.2)$ & $1(0.3)$ & $5(1.5)$ & $\ldots$ & $\ldots$ & $\ldots$ \\
\hline Transient ischemic attack & $1(0.3)$ & $\ldots$ & $1(0.3)$ & $\ldots$ & $\cdots$ & $\ldots$ \\
\hline Brain aneurysm & $1(0.3)$ & $\ldots$ & $1(0.3)$ & $\ldots$ & $\ldots$ & $\ldots$ \\
\hline Meniere's disease & $2(0.6)$ & $\ldots$ & $2(0.6)$ & $\cdots$ & $\cdots$ & $\cdots$ \\
\hline \multicolumn{7}{|l|}{ Cardiac: } \\
\hline Hypertension & $18(5.2)$ & $3(0.9)$ & $21(6.1)$ & $10(2.9)$ & $2(0.6)$ & $12(3.5)$ \\
\hline Cardiac arrhythmia & $3(0.9)$ & $\ldots$ & $3(0.9)$ & $3(0.9)$ & $\ldots$ & $3(0.9)$ \\
\hline Patent foramen ovale & $\ldots$ & $1(0.3)$ & $1(0.3)$ & $2(0.6)$ & $\ldots$ & $2(0.6)$ \\
\hline Coronary heart disease & $1(0.3)$ & $\ldots$ & $1(0.3)$ & $2(0.6)$ & $\ldots$ & $2(0.6)$ \\
\hline Cardiac valve incompetence & $\ldots$ & $\ldots$ & $\ldots$ & $1(0.3)$ & $\cdots$ & $1(0.3)$ \\
\hline Angina & $3(0.9)$ & $\ldots$ & $3(0.9)$ & $\ldots$ & $\ldots$ & $\ldots$ \\
\hline Myocardial infarction & $1(0.3)$ & $\ldots$ & $1(0.3)$ & $\ldots$ & $\ldots$ & $\ldots$ \\
\hline \multicolumn{7}{|l|}{ Psychiatric: } \\
\hline Claustrophobia & $7(2.0)$ & $5(1.5)$ & $12(3.5)$ & $5(1.5)$ & $3(0.9)$ & $8(2.3)$ \\
\hline Anxiety & $17(4.9)$ & $5(1.5)$ & $22(6.4)$ & $3(0.9)$ & $3(0.9)$ & $6(1.7)$ \\
\hline Depression & $20(5.8)$ & $9(2.6)$ & $29(8.4)$ & $1(0.3)$ & $1(0.3)$ & $2(0.6)$ \\
\hline Drug or alcohol abuse & $9(2.6)$ & $\ldots$ & $9(2.6)$ & $2(0.6)$ & $\ldots$ & $2(0.6)$ \\
\hline Panic disorder & $6(1.7)$ & $1(0.3)$ & $7(2.0)$ & $\ldots$ & $\ldots$ & $\ldots$ \\
\hline Schizophrenia & $3(0.9)$ & $\ldots$ & $3(0.9)$ & $\ldots$ & $\ldots$ & $\ldots$ \\
\hline \multicolumn{7}{|l|}{ Miscellaneous: } \\
\hline Joint surgery or trauma & $17(4.9)$ & $3(0.9)$ & $20(5.8)$ & $8(2.3)$ & $3(0.9)$ & $11(3.2)$ \\
\hline Diabetes & $\ldots$ & $\ldots$ & $\ldots$ & $1(0.3)$ & $\ldots$ & $1(0.3)$ \\
\hline Bleeding gastric or duodenal ulcer & $5(1.5)$ & $\ldots$ & $5(1.5)$ & $1(0.3)$ & . & $1(0.3)$ \\
\hline
\end{tabular}


Table 5. Reported medical conditions other than relative and absolute contraindications to diving $(n=346)$

\begin{tabular}{lcccccc}
\hline & \multicolumn{2}{c}{ Past history of disease } & & \multicolumn{2}{c}{ Presently suffers from disease } \\
\cline { 2 - 3 } \multicolumn{1}{c}{ Disease state } & Male & Female & Total & Male & Female & Total \\
\hline Tinnitus & $31(9.0)$ & $11(3.2)$ & $42(12.1)$ & $33(9.5)$ & $6(1.7)$ & $39(11.3)$ \\
Hearing loss & $8(2.3)$ & $5(1.5)$ & $13(3.8)$ & $26(7.5)$ & $3(0.9)$ & $29(8.4)$ \\
Chronic back pain & $12(3.5)$ & $2(0.6)$ & $14(4.1)$ & $10(2.9)$ & $4(1.2)$ & $14(4.1)$ \\
Arthritis & $2(0.6)$ & $\ldots$ & $2(0.6)$ & $10(2.9)$ & $2(0.6)$ & $12(3.5)$ \\
Excessive ear wax & $48(13.9)$ & $5(1.5)$ & $53(15.3)$ & $8(2.3)$ & $2(0.6)$ & $10(2.9)$ \\
Severe gastric reflux & $17(4.9)$ & $2(0.6)$ & $19(5.5)$ & $8(2.3)$ & $2(0.6)$ & $10(2.9)$ \\
Herniated spinal disc & $9(2.6)$ & $\ldots$ & $9(2.6)$ & $6(1.7)$ & $1(0.3)$ & $7(2.0)$ \\
Anemia & $1(0.3)$ & $4(1.2)$ & $5(1.5)$ & $3(0.9)$ & $2(0.6)$ & $5(1.5)$ \\
Scoliosis & $2(0.6)$ & $2(0.6)$ & $4(1.2)$ & $2(0.6)$ & $1(0.3)$ & $3(0.9)$ \\
Bronchitis & $47(13.6)$ & $24(6.9)$ & $71(20.5)$ & $3(0.9)$ & $\ldots$ & $3(0.9)$ \\
Functional bowel disease & $1(0.3)$ & $\ldots$ & $1(0.3)$ & $2(0.6)$ & $\ldots$ & $2(0.6)$ \\
Temperomandibular joint dysfunction & $\ldots$ & $\ldots$ & $\ldots$ & $2(0.6)$ & $\ldots$ & $2(0.6)$ \\
Crohn's disease & $1(0.3)$ & $\ldots$ & $1(0.3)$ & $1(0.3)$ & $\ldots$ & $1(0.3)$ \\
Pneumonia & $9(2.6)$ & $8(2.3)$ & $17(4.9)$ & $\ldots$ & $\ldots$ & $\ldots$ \\
Bowel obstruction & $6(1.7)$ & $\ldots$ & $6(1.7)$ & $\ldots$ & $\ldots$ & $\ldots$ \\
Hepatitis & $3(0.9)$ & $\ldots$ & $3(0.9)$ & $\ldots$ & $\ldots$ & $\ldots$ \\
Inguinal hernia & $2(0.6)$ & $\ldots$ & $2(0.6)$ & $\ldots$ & $\ldots$ & $\ldots$ \\
Aseptic bone necrosis & $1(0.3)$ & $\ldots$ & $1(0.3)$ & $\ldots$ & $\ldots$ & $\ldots$ \\
Polycythemia & $1(0.3)$ & $\ldots$ & $1(0.3)$ & $\ldots$ & $\ldots$ & $\ldots$ \\
& & & & $\ldots$
\end{tabular}

ing loss was also reported more than expected, with 42 divers $(12.1 \%)$ reporting this condition.

As expected, many medical conditions listed on the questionnaire had not been experienced by any of the divers. These included myasthenia gravis, stroke, multiple sclerosis, paraplegia, trigeminal neuralgia, congestive cardiac failure, mitral valve prolapse, cardiac valve stenosis, pulmonary blebs or cysts, emphysema, lung surgery, ulcerative colitis, kidney failure or transplant, osteonecrosis, limb amputation, hemophilia, leukemia, and sickle cell disease.

\section{Discussion}

The response rate of participating dive clubs was rather poor. However, the divers in this study are likely to represent experienced, recreational divers belonging to dive clubs in Australia. Each of the States and Territories was represented in approximate proportion to its population, with the exception of Queensland, which was underrepresented. This is of importance, as much of the diving in Australia is undertaken in Queensland. The finding that most divers were middle-aged males who had logged a considerable number of dives over many years is consistent with a US study of experienced, recreational divers. ${ }^{10}$ The high socioeconomic class of most divers is consistent with their ability to continue participation in this relatively expensive sport.
Although almost half of the divers were overweight, this proportion did not differ significantly $(P>.05)$ from that of the general Australian population $(45 \%)^{11}$ and is consistent with a report on other experienced divers. ${ }^{10}$ This finding has important implications. It may reflect a level of physical fitness that is suboptimal for a sport that can require considerable exertion, particularly in the event of an emergency. Also, obesity can further restrict the mobility of divers already encumbered with scuba gear. These factors may be particularly relevant to the 8 morbidly obese divers. Finally, it has been demonstrated that obesity is a risk factor for decompression sickness. ${ }^{1}$

Alcohol can directly affect diving safety in 2 ways. Clearly, diving while under the influence of alcohol is likely to increase the risk several-fold. Also, moderate or heavy alcohol intake can lead to dehydration and, in turn, an increased risk of decompression sickness. ${ }^{1,3}$ The alcohol consumption of the divers in this study is not remarkable, but the findings give no indication of the proximity of consumption to diving.

As expected, the proportion of smokers was relatively small and significantly less $(P<.01)$ than that of the Australian population (24\%). ${ }^{11}$ This finding is encouraging, as many tobacco-related respiratory diseases, including asthma and chronic obstructive airways disease, have long been considered contraindications to diving. ${ }^{1}$ A small proportion of divers did report illicit drug use, 
although, like alcohol, the proximity of drug use to diving is not known. Although only 45 divers (13.0\%) required daily medication for chronic disease, many others are likely to require medication for episodic illness (eg, asthma, allergies, and migraine).

Traditionally, there have been good theoretical reasons why many medical conditions have been contraindicated in diving. First, the subaqua environment is inherently dangerous, and any condition that might reduce the physical or mental capacity of the diver may increase these dangers significantly. Such conditions include migraine, epilepsy, seasonal allergy, asthma, cardiac disease, obesity, diabetes, hernias, and some psychiatric states. ${ }^{1-3,12,13}$ Second, some conditions are associated with an increased risk of barotrauma associated with changes in ambient pressure. Such conditions include seasonal allergies, asthma and obstructive airway diseases, pneumothorax, equalization difficulties, chronic sinusitis, middle and inner ear disease or surgery, and ocular surgery. ${ }^{1,3,13}$ Third, some conditions are associated with an increased risk of decompression illness; these include obesity, diabetes, and central nervous system and connective tissue scarring. ${ }^{1,3,5}$ This study demonstrates that many conditions within the 3 categories described are quite prevalent among experienced divers.

Few other studies have examined the prevalence of chronic disease among scuba divers. A recent Australian study by Cresp et $\mathrm{al}^{6}$ examined the health status of recently qualified divers. As expected, the subjects in that study had a similar sex ratio ( $72 \%$ male), were younger (median age, 24 years), leaner $(24.0 \%$ overweight or greater), and inexperienced ( $76 \%$ had done fewer than 20 dives). Compared with the present study, Cresp et al ${ }^{6}$ found a greater prevalence of asthma and obstructive airways disease (10.3\%) and hypertension (4.2\%) but a similar prevalence of epilepsy (1.0\%), diabetes $(0.2 \%)$, ischemic heart disease $(0.2 \%)$, and smoking (11.6\%). The authors noted that a number of divers commented on how easy it was to avoid the detection of medical conditions during the diving medical examination. Hansen et $\mathrm{al}^{10}$ reported a higher prevalence of hypertension $(9.7 \%)$ and active asthma (4.2\%) in their large study of experienced divers in the United States.

In this study, the prevalence of medical contraindications to diving is of concern. It is possible that some divers failed to disclose their conditions during the medical screening examination. ${ }^{6}$ If confirmed, this possibility would challenge the worth of the traditional screening process as an instrument for the determination of fitness to dive. A second possibility is that some divers develop contraindicated conditions as they age yet continue to dive through ignorance or neglect. In Australia, there is no requirement for repeat medical assessment for fitness to dive. This raises the question of whether medical assessment should be undertaken at regular intervals throughout a diver's career, as part of a recertification process. ${ }^{14}$

The above discussion is based on the premise that the theoretical risks of diving with medical contraindications are clinically significant. This may not be the case. Indeed, some studies have challenged traditional theory by suggesting that the risk associated with some conditions, including asthma and diabetes, may not be as great as originally thought. $8,13,15,16$ Furthermore, the available evidence shows that diving fatalities as a direct result of medical conditions are rare. An analysis of the deaths of 286 fatalities in the United Kingdom and the United States between 1990 and 1994 showed that health factors, as possible contributory causes, accounted for $2 \%$ and $5.1 \%$ of deaths, respectively. ${ }^{16}$ Australian figures appear to be higher. Of the 46 scuba diving deaths in Australia between 1993 and 1997 inclusively, 8 (17.4\%) were thought to have a possible medical contributing factor. Presumed cardiac events were considered the most common contributing medical conditions. These diving deaths included 2 cases of planned suicide. ${ }^{17-21}$

In recent years, the risks associated with many medical conditions have been reevaluated. ${ }^{5}$ This has led to policy shift among professional bodies, notably the South Pacific Underwater Medicine Society (SPUMS), regarding candidate fitness to dive $\mathrm{e}^{5,22,23}$ and a shift away from the prescription-based approach to diving fitness toward a more discretionary one. ${ }^{3,5,7}$ This would involve the medical practitioner in a risk assessment rather than a regulatory role. ${ }^{5}$ The SPUMS has proposed a draft policy statement for consideration by Standards Australia, which states: “A medical practitioner's statement of the compatibility of a candidate's health and recreational diving must include both an acknowledgment of 'health risk' and an acceptance of liability by the candidate." Should a candidate then choose to ignore the advice given, the advising physician should not be subsequently liable. ${ }^{5}$ In any event, a shift toward this discretionary approach might encourage the disclosure of existing conditions and promote a more informed discussion about the risks involved.

In this study, few divers reported other conditions that might interfere with their diving activity or be aggravated by diving. However, the reported prevalence of tinnitus and hearing difficulties is greater than expected and greater than the prevalence reported by Hansen et a ${ }^{10}$ of $8.1 \%$ and $5.1 \%$, respectively. It is conceivable that the repeated, even subclinical, aural barotrauma often experienced over a long diving career might have an etiological role in these conditions. The findings suggest that further research into the possible association be- 
tween scuba diving and chronic middle and inner ear disease is required.

This study has several limitations. The sample size was small relative to the estimated number of experienced, recreational scuba divers in Australia. Also, the recruitment of subjects from dive clubs only, the underrepresentation of Queensland clubs (and total Queensland divers), and the club response rate may have introduced selection bias. Although the questionnaire was anonymous, the results are subject to prevarication and recall bias. Finally, as a screening study examining many medical conditions, precise definitions of each condition could not be provided to the divers. This may have limited the accuracy of some responses.

This study indicates that further research is required to evaluate more clearly the risks associated with some traditionally contraindicated medical conditions. This undertaking may allow previously denied candidates the opportunity to participate in the sport with reasonable safety. If such research does confirm clinically significant theoretical risks, then the repeated reassessment of fitness to dive should be considered. It is recommended that the evolution of the traditional screening examination continue and that a greater emphasis be placed on risk assessment and candidate education. Such an evolution may require a revision of training procedures for medical practitioners planning to undertake diving medical examinations.

\section{Conclusion}

Many experienced recreational scuba divers continue to dive with medical conditions. This raises the question of nondisclosure at the initial medical screening examination or subsequent disease development. The findings may indicate either that the risks of some conditions are not of clinical significance or that repeated screening assessments are indicated. Research to assess more clearly the risks of traditionally contraindicated conditions is required, and an evolution of medical screening toward a risk assessment process is recommended.

\section{Acknowledgments}

The authors would like to thank the Pittsburgh Emergency Medicine Foundation for their financial support of this project. This Foundation played no part in the design or execution of this study and in no way controlled or influenced the final manuscript.

\section{References}

1. Edmonds C, Lowry C, Pennefather J. Diving and Subaquatic Medicine. 3rd ed. Oxford: Butterworth-Heinemann; 1992.
2. Morgan WP. Anxiety and panic in recreational scuba divers. Sports Med. 1995;20:398-421.

3. SPUMS Board of Censors. The SPUMS Recreational Diving Medical Examination. Melbourne: SPUMS; 1999.

4. Standards Australia. Training and Certification of Recreational Divers-Minimum Entry Level SCUBA Diving. Sydney: Standards Australia; 1992. AS 4005.1-1992.

5. SPUMS. 2001. SPUMS policies on medical practitioner certification for diving/diabetes and fitness for diving/asthma and fitness for diving. Available at: http://www. spums.org.au/policies.html. Accessed May 30, 2001.

6. Cresp R, Grove C, Lalor E, Valinsky L, Langton P. Health status of recreational scuba divers in Western Australia. SPUMS J. 2000;30:226-230.

7. Simpson G, Roomes D. Scuba diving medical examinations in practise: a postal survey. Med J Aust. 1999;171: 595-598.

8. Bryson P, Edge C, Gunby A, St Leger Dowse M. Scuba diving and diabetes; collecting definitive data from a covert population of recreational divers. Undersea Hyperbaric Med. 1998;25(suppl):51-52.

9. Diving in Australia. 2000. Available at: http://www. divinginaustralia.com.au/services.asp. Accessed May 22, 2000.

10. Hansen E, Fleisher J, Jackson R, Dovenbarger J, Uguccioni E, Cudahy E. 2001. Demographics and illness prevalence in recreational scuba divers. Available at: http:// www.diversalertnetwork.org/medical/articles/. Accessed May 30, 2001.

11. Australian Bureau of Statistics. National Health Survey: Summary of Results, Australia-1995. Australian Bureau of Statistics; 1995. Publication 4364.0.

12. Schioberg-Schiegnitz S. Mental fitness in technical diving for sports scuba divers. SPUMS J. 1996;26:277-280.

13. Bove AA. Medical aspects of sports diving. SPUMS J. 1996;26:247-253.

14. Elliott D. Why fitness? Who benefits from diver medical examinations? SPUMS J. 2000;30:206-209.

15. Neuman TS, Bove AA, O'Connor RD, Kelsen SG. Asthma and diving. Ann Allergy. 1994;73:344-350.

16. Health and Safety Executive, Department of Health, United Kingdom. Scuba Diving: A Quantitative Risk Assessment. Sudbury, UK: Health and Safety Executive, Department of Health; 1999.

17. Walker D. Provisional report on Australian diving-related deaths in 1993. SPUMS J. 1997;27:12-23.

18. Walker D. Australian diving-related fatalities 1994. SPUMS J. 1998;28:182-194.

19. Walker D. Australian diving-related fatalities 1995. SPUMS J. 1999;29:2-12.

20. Walker D. Australian diving-related fatalities 1996. SPUMS J. 1999;29:182-196.

21. Walker D. Australian diving-related fatalities 1997. SPUMS J. 2000;30:62-74.

22. Wendling J. Practical solutions for divers with restricted fitness to dive. SPUMS J. 2000;30:221-225.

23. Elliott D. Fit for what? What diving can be done by someone who is not perfect? SPUMS J. 2000;30:215-220. 